Article original

\title{
Les troubles cognitifs dans les syndromes cliniquement isolés: Etude Cas-Témoins
}

\author{
Cognitive impairment in clinically isolated syndromes: A control-case study
}

Mohamed Islam KEDIHA, Saliha BOULEKOUIRET, Nassima HECHAM, Sonia NOUIOUA, Lamia ALI PACHA

Service neurologie, CHU Mustapha,Alger

Auteur correspondant: kediha.islam@gmail.com Soumis le 29/03/2020 ; accepté le 04/05/2020 ; publée le 21/06/2020

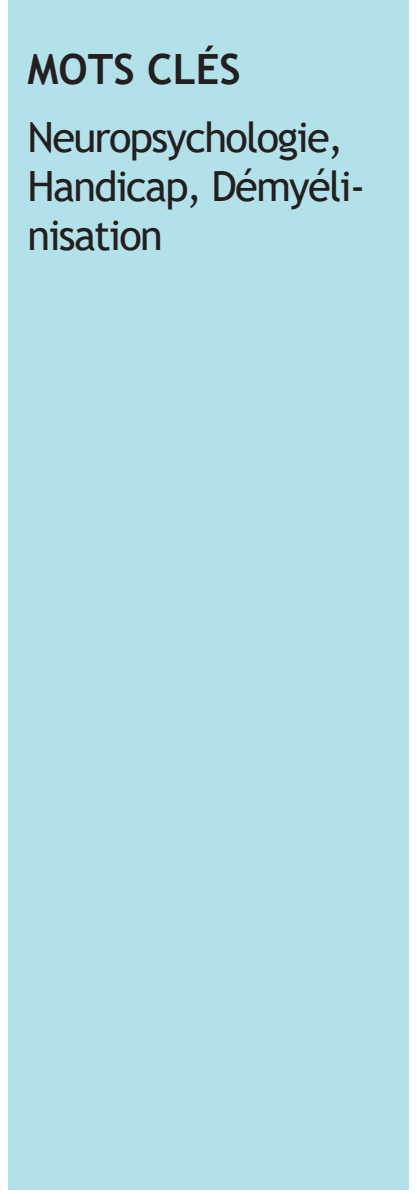

\section{Résumé}

Introduction - L'atteinte cognitive est fréquente dans la Sclérose en Plaques (SEP) mais son diagnostic se fait souvent tardivement, au stade de désinsertion sociale et professionnelle. Les études portant sur les aspects cognitifs dans les formes précoces de la SEP tels que les syndromes cliniquement isolés ( $\mathrm{SCl}$ ) et les syndromes radiologiquement isolés (SRI) sont rares.

Objectifs - Analyserles fonctions cognitives d'un premier événement démyélinisant et identifier les domainesqui seraient les plus précocement atteints.

Patients et méthodes - Le profil cognitif d'une populationhomogène de 13 patients présentant unSCl a été évalué, eta été comparé à 15 témoins sains appareillés en fonction de l'âge, du sexe et du niveau d'éducation. Une batterie de tests neuropsychologiques(BCCogSEP, batterie courte d'évaluation cognitive de la SEP), validée dans la SEP, a été utilisée. Ses composants explorent les capacités mnésiques et verbales, l'attention, la vitesse de traitement de l'information (VTI) et les fonctions exécutives.

Résultats - Lesperformances cognitives globales étaient amoindries dans le groupe $\mathrm{SCl}$, comparativement au groupe témoin. Sur les 13 patients atteints de $\mathrm{SCl}$, cinq (38\%) présentaient une altération cognitive globale qui a été objectivée par l'atteinte d'au moins deux ou trois tests de la batterie. La PASAT (Paced Auditory Serial Addition Test)était le test le plus altéré (84,6\% d'atteinte). La VTI et la mémoire de travail étaient les fonctions les plus atteintes chez les patients.

Conclusion - Les dysfonctions cognitives peuvent se voir très précocement et peuvent grever lourdement le pronostic de la SEP. 


\section{KEY WORDS}

Neuropsychology, Handicap, Demyelination

\begin{abstract}
Introduction - Cognitive impairment is common in multiple sclerosis (MS) but its diagnosis is often made late, at the stage of social and professional disinsertion. Studies of the cognitive aspects in early forms of MS such as clinically isolated syndrome (CIS) and radiologically isolated syndrome (RIS) are rare.
\end{abstract}

Objectives - To analysis the cognitive functions of a first demyelinating event and identify the areas that would be most affected early.

Patients and methods - The cognitive profile of a homogeneous population of 13 patients with a CIS was evaluated, and compared with 15 healthy controls, matched according to age, sex and level of education. A battery of neuropsychological tests (BCCogSEP, Short battery for cognitive assessment of MS) validated in MS, was used. Its components explore memory and verbal skills, attention, information processing speed (IPS) and executive functions.

Results - The overall cognitive performance was reduced in the CIS group,compared to control group. Five out of thirteen CIS patients (38\%) had an overall cognitive impairment, demonstrated by the achievement of at least two or three battery tests. The Paced Auditory Serial Addition Test (PASAT) was the most altered test $(84.6 \%$ impairment). IPS and working memory were the most affected functions in the patients.

Conclusion - Cognitive dysfunctions can be seen very early and can severely affect the prognosis of MS.

\section{Introduction}

Les troubles cognitifs (TC), avec la fatigue et la dépression, font partie des signes dits «invisibles» de la sclérose en plaques (SEP), pathologie neurologique inflammatoire très fréquente et handicapante [1]. Néanmoins, avec le regain d'intérêt récent envers ces signes et les plaintes de plus en plus fréquentes des patients en consultations spécialisées, les cliniciens font face à des symptômes non-moteurs auxquels ils n'accordent pas l'importance qu'il fallait. Le patient et le clinicien affrontent donc une double peine et une double incapacité, motrice et cognitive, qui vont contribuer à altérer la qualité de vie de façon significative, et induire la désinsertion sociale et professionnelle [2].

La fréquence des TC dans la SEP est très variable selon les séries. Elle varie entre 43 et $72 \%$ [3] ou 40 à $65 \%$ [4,5]. Ces TC peuvent se voir à tous les stades de la SEP, notamment les plus précoces, les syndromes cliniquement isolés $(\mathrm{SCl})$ voire les syndromes radiologiquement isolés (SRI) [6]. La prévalence des TC dans les $\mathrm{SCl}$ a été estimée entre 27 et $57 \%$ selon les séries [7-8-9], mais les études portant sur ces aspects cognitifs dans les formes précoces de SEP sont rares.

Dans ce travail, les performances cognitives d'un groupe de patients présentant un 1erévénementdémyélinisant(ou $\mathrm{SCl}$ ) ont été évaluées, et les principaux domaines qui seraient les plus précocement atteints dans cette affection ont été identifiés. Les données des patients ont été comparées à celles d'un groupe témoin ayant les mêmes caractéristiques épidémiologiques dans le cadre d'une étude "cas-témoins". L'hypothèse posée a été que les patients ayant unSCl,comparativement au groupe témoin, présentent une atteinte cognitive précoce et nette.

\section{Populations et Méthodes}

Type d'étude

Il s'agissait d'une étude prospective de type cas-témoin. Elle a été étalée sur une période de 6 mois (janvier 2019 et juin 2019) au niveau du service de neurologie du CHU Mustapha Pacha, Alger centre en Algérie.Les sujets des deux groupes ont signé un consentement éclairé pour participer à cette étude et ont été soumis au même protocole d'évaluation cognitive.

\section{Population}

Treize patients présentant un $\mathrm{SCl}$ ont été sélectionnés de façon prospective. Lescritères d'inclusion suivant ont été appliqués: 
âge > 18 ans, langage parlé Arabe et Français, diagnostic d'un $\mathrm{SCl}$ posé selon les critères révisés de Mc Donald 2017[10], maladie non active durant l'évaluation (pas de poussées, pas de prise de corticoïdes durant le mois précédent l'inclusion), et score fonctionnel EDSS[11] (Expanded disability status scale) entre 0 et 6.5.Les critères de non-inclusion ont été les suivants: diagnostic de SEP posé, malades en poussée, toute affection psychiatrique concomitante diagnostiquée ou évoquée, pas d'histoire de prise d'alcool ou de toxique. Le groupe contrôle $(n=15)$ a été composé de sujets sains, sans affection neurologique ou psychiatrique. Les deux groupes avaient les mêmes caractéristiques épidémiologiques (sexe, âge et niveau d'éducation ou culturel (NC)).

\section{Procédure de collecte des données}

Le diagnostic de $\mathrm{SCl}$ a été posé selon les critères révisésde $\mathrm{Mc}$ Donald [10]. Le niveau d'éducation ou NC a été évalué selon l'échelle de Barbizet et al[12].Les sept NC suivants ont été identifiés: 1:illettré;2: sait lire, écrire, compter;3: niveau fin d'étude primaire; 4 : niveau de brevet d'études de premier cycle (au total, à partir du cours préparatoire, 9 années de scolarisation) ou pour les métiers manuels niveau certificat d'aptitude professionnelle sans spécialisation; 5: niveau classe terminale (fin du deuxième cycle secondaire, 11 ou 12 années de scolarisation) ou pour les métiers manuels, niveau ouvrier ou artisan avec responsabilités techniques ou de gestion;6: niveau baccalauréat ou métiers manuels hautement qualifiés avec cursus prolongés; 7 : niveau diplôme universitaire.

L'évaluation cognitive a été réalisée avec la seule batterie francophone validée à la SEP, la BCCOgSEP (batterie courte d'évaluation cognitive de la SEP) [13].La passation de ces tests a été pratiquée par une neuropsychologue entrainée à la BCCogSEP en se basant sur une fiche d'évaluation cognitive contenant les informations du patient et du témoin (nom, prénom, âge, sexe, date de l'évaluation, date de l'événement neurologique, scores aux différents tests pratiqués). Les fonctions cognitives évaluées comprenaient la vitesse de traitement de l'information (VTI), les fonctions exécutives, la mémoire de travail et l'attention, le langage, les fonctions visio-spatiales et la flexibilité mentale. La VTI a été évaluée via le Symbol Digit Modalities Test (SDMT)[14] et la Paced Auditory Serial Addition Test (PASAT) faisant partie du MSFC (Multiple Sclerosis Functional Composite) avec le test des 25 pas ( 25 foot-walk test) et le test des neuf trous (Nine Hole Peg Test) [15]. Les fonctions exécutives ont été évaluées avec la batterie rapide d'évaluation frontale (BREF)[16]. La mémoire de travail et l'attention a été évaluée avec la PASAT et l'Empan[17] des chiffres direct et indirect. Le langage a été évalué avec le set test d'Isaacs[18]. Les fonctions visio-spatiales et la flexibilité mentale ont été évaluées avec le Trail Making Test A et B (TMT) [19]. Des seuils consensuels des normes pour chaque test ont été utilisés en fonction du sexe et du NC (moins de 12 ans ou plus de 12 ans d'études).
Analyses statistiques

Les données quantitatives et qualitatives ont été exprimées en moyenne \pm écart-type (intervalle de confiance à 95 (IC95\%)) et en nombre (pourcentage), respectivement. Les données qualitatives et quantitatives des deux groupes ont été comparées par le test de chi-deux et le test de Fisher, respectivement. Le seuil de signification statistique a été fixé à $p<0.05$, selon le logiciel Epi-info.

\section{Résultats}

L'âge moyen des patients ayant un SCl était de 29,92 ans (IC95\%: 24,82-35,03) et il était de 31,2 ans chez les témoins (IC95\%: 27,0-35,4) sans différence significative entre les deux groupes $[\mathrm{p}=0.6]$. Le niveau culturel moyen était de 5,53 chez les $\mathrm{SCl}$ (IC95\%: 4,63-6,44) et 5,93 chez les témoins (IC95\%:5,44-6,42) sans différence significative $[\mathrm{p}=0.9]$. Les données démographiques sont résumées dans le tableau 1.Les résultats des différents tests cognitifs sont résumés dans le tableau 2.

Le déficit cognitif dans les deux groupes a également été calculé en fonction du NC ( $<12$ contre de $\geq 12$ années d'études). 100\% des patients $\mathrm{SCl}$ avec moins de 12 années d'études avaient au moins un test cognitif altéré contre seulement $60 \%$ des témoins et $87,5 \%$ des patients $\mathrm{SCl}$ ayant étudié plus de 12 années contre $50,0 \%$ des témoins. Les fréquences de l'atteinte cognitive selon l'atteinte d'un, de deux ou de trois tests et plus ont été calculées. L'atteinte d'au moins trois tests d'emblée était nettement plus fréquente $(61,5 \%)$ chez les patients $\mathrm{SCl}$ que dans les témoins (26,6\%) (Tableau 3).

Au total, l'atteinte cognitive la plus fréquente dans cette série était la VTI $(84,6 \%)$, suivie de la mémoire visiospatiale $(69,2 \%)$, de la mémoire attentionnelle et de travail $(53,8 \%)$, puis du langage $(38,4 \%)$ et enfin des fonctions exécutives $(30,7 \%)$ (Figure 1$)$.

\begin{tabular}{|c|c|c|c|c|}
\hline \multicolumn{5}{|c|}{$\begin{array}{l}\text { Tableau 1. Données démographiques des deux groupes: patients ayant un syndrome } \\
\text { cliniquement isolé (SCI) et sujets témoins. }\end{array}$} \\
\hline & & $\begin{array}{l}\text { Patients SCl } \\
(n=13)\end{array}$ & $\begin{array}{l}\text { Sujets témoins } \\
\qquad(n=15)\end{array}$ & Probabilité \\
\hline \multicolumn{2}{|c|}{ Sexe (homme) } & $7(53.8 \%)$ & $6(40 \%)$ & 0,7 \\
\hline \multicolumn{2}{|c|}{ Age au diagnostic (an) } & $29,9 \pm 6,0$ & $31,2 \pm 4,0$ & 0,62 \\
\hline \multirow{3}{*}{$\begin{array}{l}\text { Niveau } \\
\text { d'éducation }\end{array}$} & Primaire & $5(38.4 \%)$ & $3(20 \%)$ & \multirow[t]{3}{*}{0,9} \\
\hline & Secondaire & $2(15.3 \%)$ & $8(53.3 \%)$ & \\
\hline & Universitaire & $6(46.1 \%)$ & $4(26.6 \%)$ & \\
\hline \multicolumn{2}{|c|}{ SCl à haut risque } & $10(77 \%)$ & $O(0 \%)$ & Non calculée \\
\hline \multicolumn{5}{|c|}{$\begin{array}{l}\text { L'âge a été exprimé en moyennetécart-type. Les données qualitatives ont été exprimées en nombre (\%). } \\
\text { Probabilité: test de chi-2 (comparaison des donnees qualitatives) et test de ficher (comparaison des donnees } \\
\text { quantitatives). }\end{array}$} \\
\hline
\end{tabular}




\begin{tabular}{|c|c|c|c|}
\hline \multicolumn{4}{|c|}{$\begin{array}{l}\text { Tableau 2. Résultats des tests des deux groupes: patients ayant un syndrome cliniquement } \\
\text { isolé (SCI) et groupe témoin. }\end{array}$} \\
\hline Tests cognitifs & $\begin{array}{l}\text { Patients SCI } \\
\qquad(\mathrm{n}=13)\end{array}$ & $\begin{array}{l}\text { Sujets témoins } \\
\quad(\mathrm{n}=15)\end{array}$ & Probabilité \\
\hline Symbol Digit Modalities Test & 30,7 & 40 & 0,70 \\
\hline Set test d'Isaac & 38,4 & 15 & 0,20 \\
\hline Empan Direct & 8 & 0 & 0,46 \\
\hline Empan Indirect & 23 & 0 & 0,04 \\
\hline Batterie rapide d'évaluation frontale & 44 & 0 & 0,03 \\
\hline Paced Auditory Serial Addition Test & 84,6 & 33,3 & 0,009 \\
\hline Trail Making Test A & 53,8 & 13,3 & 0,04 \\
\hline Trail Making Test B & 38,4 & 33,3 & 1 \\
\hline \multicolumn{4}{|c|}{ Les donńes ont été exprimées en \%. Probabilite: test de chi-2. } \\
\hline
\end{tabular}

\begin{tabular}{|c|c|c|c|}
\hline & Patients SCI (n=13) & Sujets témoins(n=15) & Probabilité \\
\hline 1 test & 15,3 & 20,0 & 0,177 \\
\hline 2 tests & 15,3 & 13,3 & 0,141 \\
\hline$\geq 3$ tests & 61,5 & 26,6 & 0,295 \\
\hline
\end{tabular}

Figure 1. Principales fonctions cognitives altérées chez les patients atteints de syndrome cliniquement isolé $(n=13)$

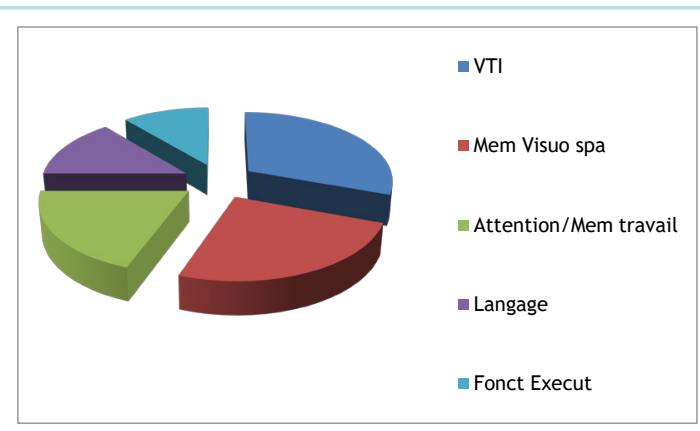

VTI: vitesse de traitement de l'information. Mem: mémoire. Visuo spa: visuo spatiale. Fonct Execut: fonction exécutive.

\section{Discussion}

Les TC dans les $\mathrm{SCl}$ peuvent grever lourdement une incapacité motricetrès invalidante. Il s'agit de symptômes très fréquents mais qui nécessitent une investigation spécifique avec des batteries neuropsychologiques de tests validés à l'exploration de la cognition dans la SEP. Ces batteries explorent la VTI, les fonctions attentionnelles, les fonctions mnésiques verbales et les fonctions exécutives. L'hypothèse posée en début de l'étude est confirmée, avec notamment des TC nets des patients SCI comparativement aux sujets témoins.

Les TC avec la fatigue et la dépression font partie des signes invisibles de la SEP pathologie neurologique inflammatoire très fréquente et handicapante (Figure 2)[1].

Figure 2.Principaux symptômes neuropsychiatriques de la sclérose en plaque. Impact sur la qualité de vie et l'emploi (D'après Penner IK, 2016[1])

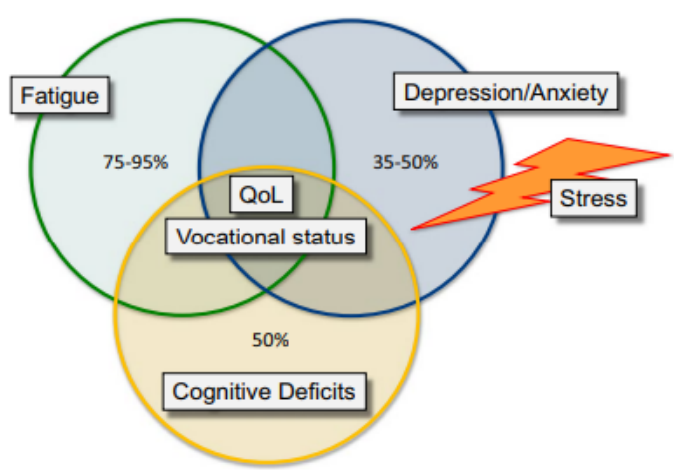

Anxiety: anxiété. Cognitive deficits: déficits cognitifs. QoL: Qualité de vie. Vocational status: statut professionnel.

Dans cette étude, les fonctions cognitives les plus altérées sont la VTI, la mémoire visuo- spatiale, la mémoire de travail et attentionnelle, le langage et les fonctions exécutives. Ceci est également décrit dans les séries Tchèque et Italienne [20, 21]. Néanmoins, il n'existait pas de différence statistiquement significative entre les deux groupes dans certaines fonctions (langage, mémoire visuo-spatiale). Les fonctions exécutivessont, par contre, altérées dans 30,7\% des cas de cette étude avec une différence significative avec le groupe témoin. Ce résultat, non rapporté dans la littérature, pourrait être expliqué par le fait que ces fonctions ne sont pas systématiquement explorées, comme dans les sériesTchèque [20], Française [22] et Autrichienne [23]. Les fluences verbales sont altérées dans $38,4 \%$ des patients $\mathrm{SCl}$ contre seulement $15,0 \%$ des sujets témoins, mais sans différence statistiquement significative. Ceci ne reflète pas les données des séries Américaine[24] et Italienne [25], dans lesquelles l'atteinte du langage est touchée de façon spécifique et précoce, notamment dans les $\mathrm{SCl}$.Le fait que $100 \%$ des patients $\mathrm{SCl}$ avec moins de 12 années d'études avaient au moins un test cognitif altéré contre seulement $60 \%$ des témoins et $87,5 \%$ des patients $\mathrm{SCl}$ ayant étudié plus de 12 années contre $50,0 \%$ des témoins supposerait que le niveau d'études bas pourrait être un facteur aggravant l'atteinte cognitive. Par ailleurs, les données de cette étude ont été comparées avec celles d'une étude Marseillaise [26] incluant 24 patients et qui avait recherché le pourcentage de patients ayant une altération d'un, de deux et de trois tests ou plus. (Tableau 4). Le pourcentage de patients avec altération de trois tests d'emblée était de $61,5 \%$ contre seule- 
ment 15,0\% pour la série Marseillaise [26] et ceci démontre une atteinte cognitive d'emblée plus sévère dans la présente étude. Ceci, d'autant plus que la même équipe a mis en évidence le fait que les $\mathrm{TC}$ dans les $\mathrm{SCl}$ avaient tendance de passer du simple au double en cinq ans [27].

\begin{tabular}{|c|c|c|}
\hline \multicolumn{3}{|c|}{$\begin{array}{l}\text { Tableau 4. Comparatif des fréquences de l'atteinte cognitive de cette étude } \\
\text { avec celles de Reuter et al. [24]. }\end{array}$} \\
\hline & Cette étude & Reuter et al.[26] \\
\hline 1 test & 15,3 & 24 \\
\hline 2 tests & 15,3 & 20 \\
\hline$\geq 3$ tests & 61,5 & 15 \\
\hline
\end{tabular}

Dans les $\mathrm{SCl}$ les TC sont considérés comme un facteur prédictif potentiel fort de progression de l'handicap, de mauvaise évolution clinique, et par conséquent ils devraient être considérés comme des marqueurs de formes agressives de cette pathologie [28]. Ceci risquerait de modifier l'attitude thérapeutique en allant vers des traitements de deuxième ligne d'emblée.

Les résultats de cette étude ont été confirmés par une étude multicentrique européenne avec plus de 16000 patients inclus [29]. Les TC avec la fatigue et le retentissement professionnel étaient présents à tous les stades de la maladie quel que soit le degré du handicap moteur et donc du score EDSS. Ceci confirme la sévérité de l'affection quand des TC s'y greffent.Dans les quelques courtes séries publiées [30,31], les TC dans les $\mathrm{SCl}$ ne sont pas évalués de façon systématique. De même, il y avait une possible évaluation au stade de syndrome radiologiquement isolé (RIS) [32].

La principale limite de la présente étude a été la faible taille de l'échantillon. Les présents résultats devraient être confirmés par une cohorte plus importante avec implication de plusieurs centres en Algérie.

\section{Conclusion}

En conclusion, la présente étude est la première à l'échelle Algérien qui explore les $\mathrm{TC}$ dans les $\mathrm{SCl}$. Ce résultat aura un impact direct sur la prise en charge thérapeutique de ce type de patients. En effet, ces patients seraient considérés comme des formes potentiellement sévères qui risqueraient d'influencer les habitudes thérapeutiques. Par ailleurs, la détection précoce de ces TC permettrait de proposer des séances de remédiation et rééducation cognitives qui ont prouvé leur efficacité dans la prise en charge dans la SEP et mettrait les cliniciens devant le challenge de pratiquer une évaluation cognitive à tous les patients.

\section{Conflits d'intérêt}

Les auteurs déclarent n'avoir aucun conflit d'intérêt.

\section{Références bibliographiques}

[1].Penner IK. Evaluation of cognition and fatigue in MS: daily practice and future directions. Acta Neurol Scand 2016; Suppl 200:19-23.

[2]. Deluca GC, Yates RL, Beale H, et al. Cognitive impairment in MS: clinical, radiologic and pathologic insights. Brain Pathol 2015; 25(1):79-98.

[3]. Chiaravallotti ND, Deluca J. Cognitive impairment in MS. Lancet Neurol 2008; 7(12):1139-51.

[4].Rao SM,Leo GJ, Ellington Let al. Cognitive dysfunction in MS, impact on employment and social functioning. Neurology 1991; 41(5):692-6.

[5].Rao SM. Neuropsychology of MS. Curr Opin Neurol 1995; 8(3):216-20.

[6].Amato MP, Hakiki B, Goretti Bet al. Association of MRI metrics and cognitive impairment in radiologically isolated syndromes. Neurology 2012; 78(5):309-14.

[7].Zipoli V, Goretti B, Hakiki B et al. Cognitive impairment predicts conversion to MS in CIS. Mult Scler 2010; 16(1):62-7.

[8].Feuillet L, Reuter F, Audoin B et al. Early cognitive impairment in patients with CIS suggestive of MS. Mult Scler 2007; 13:124-7.

[9].Potagas C, Giogkaraki E, Koutsis Get al. Cognitive impairment in different MS subtypes and CIS. J Neurol Sci 2008; 267:100-6.

[10].Jeffery A Cohen- Ectrims/Actrims MS Paris 2017.

[11].Kurtzke JF. Rating neurological impairment in multiple sclerosis: an expanded disability status scale. Neurology 1983; 33: 1444-1452.

[12].Barbizet J, Truscelli D. L'histoire du Lion. (Considérations sur la fabulation) [The story of the lion. (Considerations on deviation)]. Sem Hop. 1965;41(28):1688-1694.

[13].Dujardin K , Sockeel P, Cabaret Met al. La BCcogSEP: une batterie courte d'évaluation des fonctions cognitives destinées aux patients souffrant de sclérose en plaques [BCcogSEP: a French test battery evaluating cognitive functions in multiple sclerosis]. Rev Neurol (Paris). 2004;160(1):51-62.

[14].Deloire MSA, Bonnet MC, Salort Eet al. How to detect cognitive dysfunction at early stages of MS.Mult Scler 2006;12(4):445-452. doi:10.1191/1352458506ms1289oa

[15].Fisher JC Rudick RA, Cutter GRet al. The MS functional composite measure (MSFC): an integrated approach to MS clinical outcome assessment. Mult Scler 1999; 5:244-50.

[16].Dubois B, Slachevsky A, Litvan let al. The FAB, a frontal assessment battery at bedside. Neurology 2000; 55:1621-6.

[17].Baddeley $A D$, Thomson $N$ et Buchanan $M$. Word length and the structure of short-term memory.J verbal learning verbal behav 1975; 14(6):575-89. 
[18].Isaacs B et Kennie AT. The set test as an aid to the detection of dementia in old people.Br J Psychiatry 1973; 123:467-70.

[19].Poitsenaud j, Piette F, Sebbau Cet al. Une batterie de tests psychométriques pour l'évaluation du fonctionnement cognitif chez les sujets âgés. Rev gériatr1990; 15(12):57-68.

[20].Hyncicova E, Vyhnalek M, Kalina Aet al.Cognitive impairment and structural brain changes in patients with CIS at high risk for MS. J Neurol 2017; 264(3):482-93.

[21].Amato MP, Portaccio E, Goretti Bet al. Cognitive impairment in early stages of MS.Neurol Sci 2010 (Suppl2).S211-S214. doi:10.1007/s10072010-0376-4

[22].Feuillet L, Reuter F, Audoin B,et al. Early cognitive impairment in patients with CIS suggestive of MS. Mult Scler 2007; 13(1):124-7.

[23].Khalil M, Enzinger C, Langkammer Cet al.Cognitive impairment in relation to MRI metrics in patients with CIS. Mult Scler 2011; 17(2):173-80.

[24].Glanz BI, Holland CM, Gauthier SA et al. Cognitive dysfunction in patients with clinically isolated syndromes or newly diagnosed MS. Mult Scler 2007; 13(8):1004-10.

[25].Viterbo RG, Laffaldano P, Trojano Met al. Verbal fluencies deficits in CIS suggestive of MS. J.Neurol Sci 2013; (1-2):56-60.

[26].Reuter F, Zaaraoui W, Clespy Let al. Cognitive impairment at the onset of MS: relationship to lesion location. Mult Scler 2011; 17(6):755-8.

[27].Reuter F, Zaaraoui W, Clespy Let al. Frequency of cognitive impairment dramatically increases during the first 5 years of MS. JNeurol Neurosurg Psychiatry 2011; 82:1157-1159.

[28].Pitteri M, Romualdi C, Magliozzi Ret al. Cognitive impairment predicts disability progression and cortical thining in MS: an 8-year study. Mult Scler 2017;23(6):848-854. doi:10.1177/1352458516665496

[29]. Kobelt $\mathrm{G}$, Thomson A, Berg Jet al. New insights into the burden and costs of MS in Europe. Mult Scler 2017; 23(8):1123-36.

[30].Feinstein A, Kartsounis LD, Miller DHet al.Clinically isolated lesions at the type seen in MS: a cognitive, psychiatric and MRI follow up study. J Neurol Neurosurg Psychiatry 1992; 55(10):869-76.

[31].Achiron A et Barak Y. Cognitive impairment in probable MS. J Neurol Neurosurg Psychiatry 2003; 74(4):443-6.

[32].Lebrun C, Blanc F, Brassat Det al. Cognitive function in RIS. Mult Scler 2010; 16(8):919-25. 